\title{
Frecuencia de síndrome metabólico en niños y adolescentes de una escuela de Asunción de acuerdo a distintas definiciones
}

\author{
Frequency of metabolic syndrome in children and \\ adolescents of a school in Asunción according to different \\ definitions
}

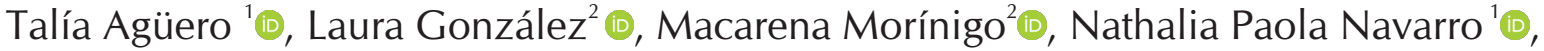

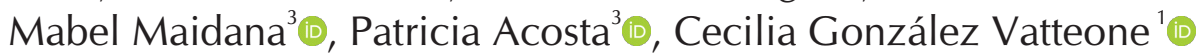

\section{RESUMEN}

Introducción: Hasta la fecha no se ha podido establecer un consenso para definir de forma universal el Síndrome Metabólico (SM) en niños y adolescentes. En Paraguay, no ha sido documentada la variación de la frecuencia de SM en niños y adolescentes de acuerdo a las diferentes definiciones propuestas en la literatura. Objetivo: Determinar la frecuencia de Síndrome Metabólico en niños y adolescentes de una escuela de la ciudad de Asunción utilizando 3 diferentes definiciones. Materiales y Métodos: Se realizó un estudio de corte transversal en niños y adolescentes (9-13 años). Se recolectaron datos demográficos y mediciones antropométricas. Se extrajeron muestras de sangre en ayunas. Se analizó el colesterol total, triglicéridos, HDL-colesterol, LDLcolesterol y se midió la presión arterial. La frecuencia de SM se estimó a partir de las definiciones modificadas de la Federación Internacional de Diabetes (IDFmod), Programa Nacional de Educación sobre Colesterol (NCEPmod) y De Ferranti. El protocolo de estudio fue aprobado por el comité de ética de la FCQ-UNA Cod 340/17. Resultados: Se analizaron datos de 76 niños y adolescentes (el 56\% de sexo masculino), cuya edad promedio fue de 10,5 $\pm 1,1$ años. El $54 \%$ presentó sobrepeso y obesidad. La frecuencia de SM fue 5,26\% según IDFmod,

\begin{abstract}
Introduction: To date it has not been possible to establish a consensus to universally define the Metabolic Syndrome (MS) in children and adolescents. In Paraguay, the variation in the frequency of MS in children and adolescents has not been documented according to the different definitions proposed in the literature. Objective: To determine the frequency of Metabolic Syndrome in children and adolescents of a school in the city of Asunción using 3 different definitions. Materials and Methods: This was a cross-sectional study that was carried out in children and adolescents (9-13 years). Demographic data and anthropometric measurements were collected. Fasting blood samples were drawn. Total cholesterol, triglycerides, HDL-cholesterol, LDL-cholesterol were analyzed and blood pressure was measured. The frequency of MS was estimated from the modified definitions of the International Diabetes Federation (IDFmod), National Cholesterol Education Program (NCEPmod) and De Ferranti. The study protocol was approved by the ethics committee of the FCQ-UNA Cod 340/17. Results: Data from 76 children and adolescents (56\% male), whose average age was $10.5 \pm 1.1$ years, were analyzed. $54 \%$ were overweight and obese. The frequency of MS was $5.26 \%$ according to IDFmod, $7.89 \%$ according to
\end{abstract}

${ }^{1}$ Universidad Nacional de Asunción, Facultad de Ciencias Químicas, Departamento de Investigación de Bioquímica Clínica. San Lorenzo, Asunción.

${ }^{2}$ Universidad Nacional de Asunción, Facultad de Ciencias Químicas, Departamento de Investigación de Nutrición. San Lorenzo, Asunción.

${ }^{3}$ Universidad Nacional de Asunción, Facultad de Ciencias Químicas, Departamento de Investigación de Farmacia. San Lorenzo, Asunción.

Correspondencia: Cecilia González Vatteone Correo: cgonzalez@qui.una.py

Conflictos de interés: Los autores declaran no presentar conflicto de interés.

Recibido: 24/01/2021 Aceptado:15/02/2021

DOI: https://doi.org/10.31698/ped.48012021005

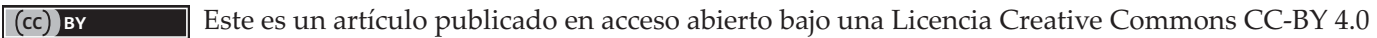


7,89\% según NCEPmod y 22,37\% de según De Ferranti. Conclusión: La frecuencia de SM fue mayor al emplear la definición propuesta por De Ferranti. Utilizar definiciones cuyos puntos de corte son más estrictos podría tener un efecto en términos preventivos al detectar precozmente a los niños y adolescentes en riesgo.

Palabras Clave: Síndrome Metabólico, niños, adolescentes.
NCEPmod and $22.37 \%$ according to De Ferranti. Conclusion: The frequency of MS was higher when using the definition proposed by De Ferranti. Using definitions whose cut-off points are stricter could have a preventive effect by early detection of at-risk children and adolescents.

Key Words: Metabolic Syndrome, children, adolescents.

\section{INTRODUCCIÓN}

El Síndrome metabólico (SM) hace referencia a una condición clínica que implica un conjunto de factores de riesgo (obesidad abdominal, presión arterial elevada, dislipidemias, resistencia a la insulina e intolerancia a la glucosa) que pueden causar enfermedades cardiovasculares y/o Diabetes Mellitus tipo $2^{(1-3)}$.

En las últimas décadas se ha observado un aumento en la prevalencia obesidad infantil y la aparición de enfermedades metabólicas a edades cada vez más tempranas. La evidencia indica que, los niños que se posicionan en los percentiles superiores de una determinada trayectoria de riesgo, tienden a mantenerse en la misma hasta la adultez, lo que podría predecir mayor morbimortalidad en edades posteriores $^{(4-9)}$.

Si bien para la población adulta existe mayor consenso sobre la definición del SM, en la edad pediátrica, no se aplica una definición única ${ }^{(10,11)}$. En niños y adolescentes, las definiciones para diagnosticar SM son aún controversiales en términos de relevancia clínica, puntos de corte utilizados, edad a partir de la cual puede ser diagnosticado y cantidad de factores de riesgo que se consideran necesarios para su diagnóstico ${ }^{(1,12-14)}$.

En niños y adolescentes, realizar la evaluación y el diagnóstico del SM, resulta difícil, debido a la compleja fisiología de los mismos a causa del crecimiento y desarrollo y la variabilidad biológica de cada organismo. Ante este escenario, es necesario establecer una definición que permita realizar un tamizaje adecuado de aquellos que presentan más factores de riesgo para el síndrome ${ }^{(6)}$.
A lo largo de los años varias definiciones se han propuesto y las más frecuentemente utilizadas han sido las del Grupo de Consenso Internacional de la Federación de Diabetes (IDF), Programa Nacional de Educación sobre el Colesterol-Panel de Tratamiento de Adultos (NCEP-ATP) modificado por Cook et al., la de Ferranti y la de Jolliffe y Janssen ${ }^{(12-14)}$.

A nivel regional un estudio realizado por Pierlot et al. reportó la presencia variable de SM y sus componentes en poblaciones de niños y adolescentes de América, ubicando a Paraguay entre los países con prevalencia inferior al 6\%. En el plano local RiverosSasaki et al. (2012) reportaron una frecuencia mínima de SM $(0,8 \%)$ en adolescentes, mencionando a la presión arterial y HDL-colesterol alterados como los componentes predominantes. A la fecha, no se han hallado estudios más recientes relacionados al tema $a^{(1,15,16)}$.

Los niños y los adolescentes son una de las poblaciones más expuestas a situaciones de malnutrición (por déficit y por exceso), desde el punto de vista de salud pública este grupo es de gran interés, pues durante esta etapa se adquieren y consolidan las actitudes y comportamientos relativos al cuidado de la salud que permanecerán a lo largo de la vida, por lo tanto, realizar un tamizaje adecuado podría permitir la intervención a edades tempranas a fin de modificar la trayectoria de riesgo. Es por ello que, ante la necesidad de definir criterios para el diagnóstico del SM en niños y adolescentes, se buscó determinar la frecuencia de SM utilizando diferentes definiciones en una población pediátrica de 9 a 13 años de edad. 


\section{MATERIALES Y MÉTODOS}

Se realizó un estudio observacional descriptivo de corte transversal con datos secundarios. Los sujetos de estudio fueron niños y adolescentes de una escuela situada en la zona de la ribera del río Paraguay en la ciudad de Asunción. El reclutamiento se realizó en el mes de junio del año 2017 en el marco de un proyecto institucional y el presente estudio, fue llevado a cabo entre los meses de agosto a setiembre del mismo año. Para la selección de los participantes se utilizó un muestreo no probabilístico de casos consecutivos.

Los criterios de elegibilidad consideraban a todos los niños y adolescentes de 9 a 13 años, que se encontraban cursando el $4^{\circ}$, $5^{-}$o $6^{-}$grado de la Educación Escolar Básica, de los turnos mañana y tarde, cuyos padres firmaron un consentimiento informado. Se excluyó a los participantes que manifestaron presentar patologías como Diabetes Mellitus tipo 1, enfermedades metabólicas raras o que se encontraban consumiendo medicamentos que pudieran alterar el metabolismo, además se excluyó a los que presentaban datos incompletos.

En el proyecto institucional se establecieron procedimientos para la recolección de los datos. Primeramente se codificó a cada participante y se registraron datos demográficos (sexo y edad). Para la obtención de los datos clínicos-laboratoriales se estableció un circuito de medición, donde se registró la Presión Arterial (PA), seguida de la toma de muestra de sangre, mediciones antropométricas y aplicación de una encuesta estandarizada.

Se realizó una única medición de la PA sistólica y diastólica, luego de verificar que los participantes no tuvieran ganas de ir al baño y que permanecieran sentados, en reposo durante al menos 5 minutos. Se utilizó un tensiómetro aneroide, pediátrico y de adulto, de la marca OMRON, que fue colocado sobre la arteria braquial derecha. Dependiendo de la circunferencia braquial de cada participante se utilizó el brazalete pediátrico de 17 a $22 \mathrm{~cm}$ o de adultos de 22 a $42 \mathrm{~cm}$. La clasificación de los percentiles de PA se determinó usando tablas normativas para la edad, género y estatura de cada niño, del Programa Nacional de Prevención Cardiovascular del Ministerio de Salud Pública y
Bienestar Social (MSPBS) de Paraguay, basada en la propuesta de la Academia Americana de Pediatría.

Las determinaciones de glucosa, triglicéridos, Colesterol total y HDL-colesterol fueron realizadas por profesionales bioquímicos, utilizando muestras de suero obtenidas por centrifugación a partir de muestras de sangre extraídas por venopunción. Antes de la toma de muestra, se verificó que cada participante cumpliera con el ayuno mínimo de 10 a 12 horas y se descartó la presencia de fiebre $u$ otros síntomas de infección aguda. Las muestras se analizaron utilizando un equipo semi-automatizado de marca BioSystems, modelo BTS 350. Se emplearon kits comerciales de la marca Human Diagnostics Worldwide (Alemania) basados en técnicas colorimétricas enzimáticas.

Las mediciones antropométricas fueron realizadas por nutricionistas previamente entrenadas, quienes recolectaron los datos por duplicado, utilizando instrumentos calibrados. La medición del peso corporal se realizó con una balanza electrónica portátil (Omron HBF-510LA). La talla se determinó con un estadiómetro portátil (Seca 213). La cintura fue definida como la circunferencia mínima entre la cresta iliaca y la caja torácica y se midió con una cinta métrica flexible y no elástica (Lufkin W606PM). Se utilizaron las mediciones de peso y talla y se calcularon los puntajes estandarizados del Índice de Masa Corporal para la edad (IMC/E), a través del software WHO Anthro Plus versión 1.0.4 (2007). El estado nutricional se determinó empleando los puntos de corte de la Organización Mundial de la Salud (OMS), 2006.

La presencia de Síndrome Metabólico se evaluó utilizando la definición del Programa de Educación sobre Colesterol modificado por Cook (NCEP-ATP III), la definición de De Ferranti et al. y la de IDF modificada para adolescentes, diferenciándose entre sí por la cantidad de factores mínimos considerados para diagnosticar a un paciente, así como por los puntos de corte de cada uno de ellos (Tabla 1) $)^{(10,12-14,17,18)}$. 
Tabla 1. Definiciones de Síndrome Metabólico utilizadas en el estudio.

\begin{tabular}{llll}
\hline & NCEP-ATP III & De Ferranti & IDF \\
\hline Definición de SM & 3 o más factores de riesgo & 3 o más factores de riesgo & $\begin{array}{l}\text { Obesidad central }+ \\
\text { 2 factores de riesgo }\end{array}$ \\
\hline Circunferencia de cintura & $\begin{array}{l}\geq 0 \text { percentil según } \\
\text { edad y sexo }\end{array}$ & $\begin{array}{l}>75 \text { percentil según } \\
\text { edad y sexo }\end{array}$ & $\begin{array}{l}\geq 90 \text { percentil según } \\
\text { edad y sexo }\end{array}$ \\
\hline Glucosa en ayunas & $\geq 110 \mathrm{mg} / \mathrm{dL}$ & $\geq 110 \mathrm{mg} / \mathrm{dL}$ & $\geq 100 \mathrm{mg} / \mathrm{dL}$ \\
\hline Triglicéridos & $\geq 110 \mathrm{mg} / \mathrm{dL}$ & $\geq 100 \mathrm{mg} / \mathrm{dL}$ & $\geq 150 \mathrm{mg} / \mathrm{dL}$ \\
\hline HDL-colesterol & $\leq 40 \mathrm{mg} / \mathrm{dL}$ & $<50 \mathrm{mg} / \mathrm{dL}$ & $<40 \mathrm{mg} / \mathrm{dL}$ \\
\hline Presión arterial Sistólica & $\geq 90$ percentil según & $\geq 90$ percentil según & $\geq 130 \mathrm{mmHg}$ \\
& edad, sexo y altura & edad, sexo y altura & \\
\hline Presión arterial Diastólica & $\geq 90$ percentil según & $\geq 90$ percentil según & $\geq 85 \mathrm{mmHg}$ \\
& edad, sexo y altura & edad, sexo y altura & \\
\hline
\end{tabular}

NCEP ATP III: National Cholesterol Education Program Adult Treatment Panel III.

IDF: Federación Internacional de la Diabetes (IDF, por sus iniciales en inglés).

Para la gestión de datos, se procedió a depurar la base de datos utilizando los criterios de selección establecidos, se comprobó la consistencia y plausibilidad de los valores obtenidos y se inspeccionó la distribución de las variables utilizando gráficos de distribución diagnóstica y estimación univariada de la densidad, además de las pruebas de normalidad de asimetría y curtosis y el test de Kolmogorov-Smirnov. Para resumir los datos se utilizaron medidas de tendencia central y dispersión. Las variables continuas con distribución normal se presentaron como medias y desviación estándar y las continuas sin distribución normal se resumieron como mediana y rangos intercuartílicos. Finalmente, las variables categóricas se presentaron como frecuencias absolutas y porcentaje. Para el análisis de los datos se utilizó el software estadístico Stata12® (StataCorp LP, College Station, Texas 77845, Estados Unidos).

\section{RESULTADOS}

Participaron del estudio 76 niños. En la Tabla 2 se presentan las características demográficas de los participantes del estudio. El 56,6\% de los niños pertenecían al sexo masculino y la edad promedio fue de $10,5 \pm 1$ años.

Tabla 2. Características demográficas de los niños y adolescentes de una escuela de Asunción (n=76).

\begin{tabular}{lcclc}
\hline Características demográficas & $\mathrm{n}(\%)$ & \multicolumn{3}{c}{ IC 95\% } \\
\hline Sexo & & & & \\
$\quad$ Femenino & $33(43,4)$ & 32,3 & - & 54,5 \\
$\quad$ Masculino & $43(56,6)$ & 45,5 & - & 67,6 \\
$\quad$ Edad (años) & & 10,3 & - & 10,8 \\
$\quad$ Categorías de edad & $10,5(1,1)$ & & & \\
$\quad<11$ años & & 50,9 & - & 72,7 \\
$\quad \geq 11$ años & $47(61,8)$ & 27,3 & - & 49,1 \\
Grado escolar & $29(38,2)$ & & & \\
$\quad 4^{\circ}$ grado & & 18,7 & - & 39,1 \\
$5^{\circ}$ grado & $22(28,9)$ & 27,3 & - & 49,1 \\
$6^{\circ}$ grado & $29(38,2)$ & 22,3 & - & 43,5 \\
\hline
\end{tabular}

${ }^{a}$ Valores presentados como media \pm desviación estándar 
En la Tabla 3 se resumen los datos antropométricos y el estado nutricional de los participantes del estudio.
Se destaca el hallazgo de $23,7 \%$ de participantes con sobrepeso y $30,3 \%$ con obesidad.

Tabla 3. Características nutricionales de los niños y adolescentes de una escuela de Asunción (n=76).

\begin{tabular}{|c|c|c|c|c|}
\hline Características nutricionales & $\bar{X}$ o n (DE o \%) & \multicolumn{3}{|c|}{ IC 95\% } \\
\hline \multicolumn{5}{|l|}{ Antropométricas, $\bar{X}(D E)$} \\
\hline Peso $(k g)$ & $42,8(11,7)$ & 40,1 & - & 45,5 \\
\hline Talla $(\mathrm{cm})$ & $144(8)$ & 142 & - & 146 \\
\hline $\mathrm{IMC}(\mathrm{kg} / \mathrm{m} 2)$ & $20,4(4,3)$ & 19,4 & - & 21, \\
\hline Circunferencia de cintura $(\mathrm{cm})$ & $69,9(11,6)$ & 67,2 & - & 72,5 \\
\hline Puntaje z IMC/Edad a & $1,0(1,3)$ & 0,7 & - & 1,3 \\
\hline Puntaje z Talla/Edad a & $0,3(1,0)$ & 0,1 & - & 0,5 \\
\hline \multicolumn{5}{|l|}{ Estado nutricional, $n(\%)^{b}$} \\
\hline Desnutrición & $2(2,6)$ & $-1,0$ & - & 6,2 \\
\hline Riesgo de desnutrición & $4(5,2)$ & 0,2 & - & 10,2 \\
\hline Normal & $29(38,2)$ & 27,3 & - & 49,1 \\
\hline Sobrepeso & $18(23,7)$ & 14,1 & - & 33,3 \\
\hline Obesidad & $23(30,3)$ & 20,0 & - & 40,6 \\
\hline
\end{tabular}

IMC: Índice de masa corporal. X (DE): promedio \pm desviación estándar.

a Puntaje Z basado en los estándares de crecimiento de la Organización Mundial de la Salud; OMS 2007.

${ }^{b}$ Estado nutricional: Desnutrición $<-2 D E$, Riesgo de desnutrición $\geq-2$ y $<-1 D E$, Normal $\geq-1$ y $\leq 1 D E$, Sobrepeso $>1$ y $\leq 2 D E$, obesidad $>2 D E$.

En la Tabla 4 se presentan los datos clínicolaboratoriales de los participantes del estudio. Entre los resultados se destaca que el $17,1 \%$ presentó niveles de Colesterol HDL y triglicéridos por encima del punto de corte.

Tabla 4. Características clínicas-laboratoriales de los 76 niños y adolescentes de una escuela de Asunción.

\begin{tabular}{|c|c|c|}
\hline Características clínicas & Mediana (P25-P75) ${ }^{\mathrm{a}}$ & $\begin{array}{c}\text { Por encima del punto de corte } \\
\text { n (\%) }\end{array}$ \\
\hline Colesterol-HDL (mg/dL) & $43(3$ & $13(17,1)$ \\
\hline Triglicéridos (mg/dL) & 4) & (1) \\
\hline Glicen & & - \\
\hline rial sistólica $(\mathrm{mmHg})$ & ) & $7(9,2)$ \\
\hline Presión arterial diastólica $(\mathrm{mmHg})$ & $60(60-70)$ & $6(7,8)$ \\
\hline \multicolumn{3}{|c|}{${ }^{a}$ Valores expresados como mediana y Percentiles 25 y 75} \\
\hline \multicolumn{3}{|c|}{$\begin{array}{l}{ }^{b} \text { Puntos de corte: HDL-colesterol } \leq 5 \text { percentil [Asociación Americana de Pediatría: niñas (de } 5 \text { a } 9 \text { años: } \leq 36 \\
\text { mg/dL, de } 10 \text { a } 14 \text { años: } \leq 37 \mathrm{mg} / \mathrm{dL} \text { ) y niños (de } 5 \text { a } 9 \text { años: } \leq 38 \mathrm{mg} / \mathrm{dL} \text {, de } 10 \text { a } 14 \text { años: } \leq 37 \mathrm{mg} / \mathrm{dL} \text { )]. } \\
\text { Triglicéridos } \geq 95 \text { percentil [Asociación Americana de Pediatría: niñas (de } 5 \text { a } 9 \text { años: } \geq 120 \mathrm{mg} / \mathrm{dL} \text {, de } 10 \text { a } 14 \\
\text { años: } \geq 120 \mathrm{mg} / \mathrm{dL} \text { ) y niños (de } 5 \text { a } 9 \text { años: } \geq 85 \mathrm{mg} / \mathrm{dL} \text {, de } 10 \text { a } 14 \text { años: } \geq 111 \mathrm{mg} / \mathrm{dL} \text { )]. Glicemia en ayunas } \geq \\
100 \mathrm{mg} / \mathrm{dL} \text { [Federación Internacional de Diabetes]. Presión arterial sistólica y diastólica } \geq 90 \text { percentil [Ferranti et } \\
\text { al.: niñas } 9 \text { años: } 116 / 75 \mathrm{mmHg}, 10 \text { años: } 118 / 76 \mathrm{mmHg}, 11 \text { años } 119 / 77 \mathrm{mmHg}, 12 \text { años: } 121 / 78 \mathrm{mmHg}, 13 \text { años } \\
123 / 79 \mathrm{mmHg} \text { niños de } 9 \text { años: } 117 / 76 \mathrm{mmHg}, 10 \text { años: } 119 / 77 \mathrm{mmHg}, 11 \text { años: } 120 / 78 \mathrm{mmHg}, 12 \text { años: } 123 / 78 \\
\mathrm{mmHg}, 13 \text { años: } 125 / 79 \mathrm{mmHg} \text { ] }\end{array}$} \\
\hline
\end{tabular}


Con relación a los factores de riesgo considerados (Figura 1), empleando la definición de la IDF se obtuvieron niveles alterados de HDL-colesterol en el $31,58 \%$ de los niños, de triglicéridos en el 3,95\% y de circunferencia de cintura en el 22,37\%, mientras que no se observaron niños con alteraciones de glicemia o de presión arterial. Utilizando la definición del NCEP-
ATP III se obtuvieron niveles alterados de HDLcolesterol en el 35,53\% de los niños, de triglicéridos en el 19,74\%, de circunferencia de cintura n el 22,3\%, de presión arterial sistólica en el 9,21\% y de presión arterial diastólica en el 7,89\%, no observándose niveles alterados de valores de glicemia.



Figura 1. Niveles de factores de riesgo presentes en los niño y adolescentes según las definiciones empleadas $(\mathrm{n}=76)$.

La aplicación de la definición de De Ferranti, arrojó alteraciones en los niveles de colesterol-HDL en el 75\% de los niños, en los niveles de triglicéridos en el $28,95 \%$, circunferencia de cintura en el $40 \%$, mientras que para la presión arterial sistólica y diastólica los valores fueron los mismos que los observados utilizando la definición de la NCEP-ATP III. Con esta definición tampoco se observaron alterados los niveles de glicemia.

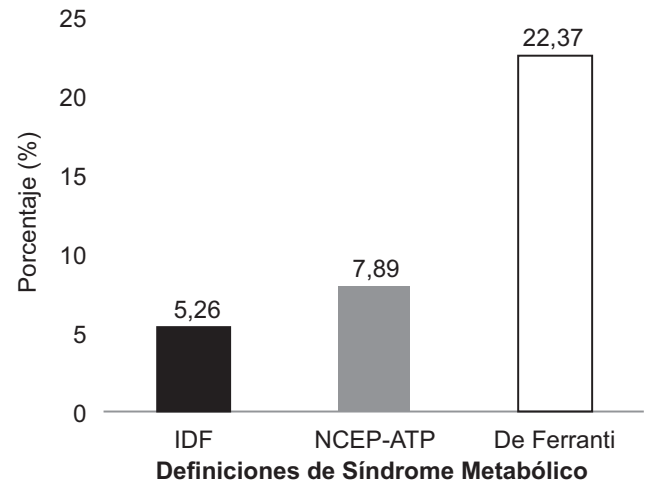

En cuanto a la frecuencia de Síndrome Metabólico según cada definición utilizada (Figura 2) se obtuvo una frecuencia de 5,26\% utilizando la definición de la IDF modificada para adolescentes, 7,89\% empleando la definición del NCEP-ATP III y 22,37\% utilizando la definición de De Ferranti.
Figura 2. Frecuencia del Síndrome Metabólico en 76 escolares, según IDF, NCEP-ATP III y De Ferranti. 
Al clasificar a los niños con SM de este estudio, según el estado nutricional, empleando tanto la definición de la IDF como la del NCEP-ATP III se observó que el 100\% de los niños con SM tenían exceso de peso (sobrepeso u obesidad), utilizando la definición de De Ferranti se observó que el 94,1\% de los niños con SM tenían exceso de peso y el 5,9\% normopeso.

\section{DISCUSIÓN}

Según el IMC/E de los niños versus los estándares de referencia de OMS, el 30,3\% de los participantes de este estudio presentó obesidad y el 23,7\% presentó sobrepeso. Estos valores son mayores a lo reportado por el Sistema de Vigilancia Alimentaria y Nutricional (SISVAN) del Ministerio de Salud Pública y Bienestar Social en el año 2016 (obesidad $10,7 \%$ y sobrepeso $20,2 \%)^{(19-21)}$.

Un estudio realizado por Vio del R et al. (2017), en niños chilenos de 8 a 11 años, reportó una frecuencia similar a la obtenida en este estudio (30,3\%). Según los datos de la Junta Nacional de Auxilio Escolar y Becas (JUNAEB) ${ }^{(22)}$, en el año 2015, la obesidad de esta población de niños chilenos fue del $24,2 \%$, lo que indica el aumento de prevalencia de la obesidad infantil ${ }^{(23)}$.

Las frecuencias de SM obtenidas en esta investigación, aplicando las distintas definiciones, se encuentran contenidas en el intervalo de resultados obtenidos en un estudio realizado por RamírezVélez et al., en 675 niños y 1.247 adolescentes de 9 a 17 años, de escuelas públicas de Colombia, donde las frecuencias variaron de 0,3 a $11 \%$ utilizando diferentes definiciones operacionales de $\mathrm{SM}^{(24)}$.

Al utilizar la definición de SM de acuerdo a la versión modificada del NCEP-ATP III, Barbalho et al., hallaron que en un grupo de niños brasileros de 6 a 10 años la frecuencia de SM fue 17,8\%, la cual es mayor a la observada en este estudio $(7,89 \%)$, las discrepancias podrían explicarse por las variaciones en las edades poblacionales, hábitos nutricionales, actividad física, entre otras ${ }^{(2)}$.

Guzmán et al., realizaron un estudio en 225 niños Mexicanos de 6 a 12 años de los cuales 106 eran obesos y 119 tenían peso saludable. Observaron una gran diferencia en la frecuencia de SM entre los dos grupos de niños, utilizando la definición de la IDF, con $44,3 \%$ de frecuencia de SM para el grupo de niños obesos, frente a $0,84 \%$ en el grupo de niños de peso saludable. Frente a estos resultados, de los niños con SM del presente estudio, el 100\% presentó exceso de peso (sobrepeso u obesidad) empleando las definiciones de la IDF y el NCEP-ATP III, mientras que según la definición de De Ferranti el valor fue de $94,11 \%$. Estos resultados indican el papel relevante de la obesidad, por lo cual es necesario una detección precoz de la misma y la toma de medidas de intervención para reducir los riesgos de sufrir alteraciones metabólicas ${ }^{(19)}$.

Si bien la frecuencia de SM encontrada en el grupo de estudio es baja, debido a que se trata de una evaluación temprana de los factores de riesgo, se considera relevante teniendo en cuenta que los trastornos metabólicos a que inician de manera precoz tienden a continuar hasta la adultez y representan un mayor riesgo de desarrollo de trastornos cardio-metabólicos y de otras patologías asociadas a la disminución de la esperanza y calidad de vida. Es así como la detección precoz adquiere gran relevancia, pues permitiría implementar acciones individuales o poblaciones de manera oportuna, con el objetivo de promover un estilo de vida saludable desde la infancia y por ende disminuir el riesgo de morbimortalidad a futuro ${ }^{(9)}$.

Debido a que aún no se ha establecido una única definición de diagnóstico de SM y sobre todo, que al estudiar poblaciones pediátricas, se aplican adecuaciones de las utilizadas para adultos, se requieren de más estudios que permitan llegar a un consenso respecto a los factores de riesgo y los puntos de corte a ser considerados para establecer la definición estandarizada.

En este estudio se observó mayores frecuencias de factores de riesgo de SM con la definición de De Ferranti, en comparación con las observadas con las definiciones de la IDF y el NCEP-ATP III. Esto se debería a que a pesar de que las tres consideran los mismos factores de riesgo, tienen puntos de corte diferentes entre sí. 
El estudio de Barbalho et al., reportó que los factores de riesgo de mayor prevalencia fueron la circunferencia de cintura con valores iguales o mayores al percentil 90 en el 93,8\% de su población de estudio, utilizando la definición del NCEP-ATP III, valor mucho mayor al observado en el este estudio, con 22,37\%. En el caso de los niveles de HDL-colesterol, el 42,3\% del grupo de niños brasileros presentó niveles inferiores a los $40 \mathrm{mg} / \mathrm{dL}$, similar a lo observado en este estudio con 35,53\% según el NCEP-ATP III ${ }^{(25)}$.

El NCEP-ATP III posee el mismo punto de corte que la IDF para la circunferencia de cintura. Esta definición no condiciona el diagnóstico del SM a la presencia obligatoria de ninguno de sus factores de riesgo, sin embargo, los individuos deben reunir al menos tres de los mismos para ser diagnosticados, aun así NCEP-ATP III reconoce a la obesidad como un factor relevante en sujetos con SM o riesgo ${ }^{(10,13,17)}$.

En el caso de la definición de De Ferranti, considera a la circunferencia de cintura como factor de riesgo cuando sus valores son superiores al percentil 75 según edad y sexo correspondiente, un punto de corte muy diferente a los de la IDF y el NCEP-ATP III, este podría ser el principal causante de la diferencia de casi cuatro veces mayor de la frecuencia deSM observada con esta definición.

Pretto et al. ${ }^{(26)}$, estudiaron la frecuencia de los factores de riesgo de SM en 616 niños de 8 años de edad del Sur de Brasil y reportaron que 20,7\% de los niños tenía circunferencia de cintura con valores mayores al percentil 90 . Este resultado es similar a lo observado en este estudio con las definiciones de la IDF y el NCEP-ATP III.

Las frecuencias de SM en este estudio fueron similares utilizando las definiciones de la IDF modificada para adolescentes y el NCEP-ATP III modificado por Cook, y fue mucho mayor empleando la definición de De Ferranti, las frecuencias de factores de riesgo variaron entre las tres. Esto se debería a las diferencias en los puntos de corte de las mismas.

Vanlancker et al. ${ }^{(13)}$, realizó un estudio en 1004 niños y adolescentes de ciudades europeas, reportando desde 1,6\% hasta 3,8\% de individuos con SM, utilizando cinco definiciones diferentes, al comparar a los diagnosticados, destacó que no se lograba clasificar a los mismos sujetos, hipotetizando que se debería a las diferencias en los puntos de corte utilizados, lo cual representa un desafío para el diagnóstico certero.

La definición del NCEP-ATP III para SM establecida para adultos, es la más utilizada por su sencillez, aun así, es utilizada en la mayoría de los estudios realizados en población pediátrica con modificaciones realizadas por Cook et al., ya que no existe una definición para los mismos y esta definición se vuelve conveniente por sus factores de riesgo medidos por edad y sexo correspondiente.

En el caso de la definición de Jolliffe y Janssen, esta establece puntos de corte por edad y sexo en individuos de 12 a 19 años $^{(13)}$, y el grupo etario de este estudio incluye niños desde 9 años, y en mayor proporción 10 y 11 años, motivo por el cual no se utilizó esta definición.

\section{CONCLUSIONES}

En la población pediátrica ( 9 a 13 años) de una escuela de Asunción las frecuencias de factores de riesgo de $\mathrm{SM}$ variaron según la definición empleada, debido a los distintos puntos de corte utilizados. A pesar de que el criterio NCEP-ATP III es ampliamente utilizado, desde una mirada preventiva, se recomienda la definición propuesta por De Ferranti por su capacidad de detectar mayor frecuencia de SM, considerando que el diagnóstico precoz permitirá una intervención oportuna en la población infantil.

Aspectos éticos: El protocolo del presente estudio fue aprobado por el CEI de la FCQ-UNA, con el Código N³40/17.

Fuentes de financiamiento: Los datos secundarios utilizados en el presente estudio están enmarcados en el Proyecto Institucional “Evaluación integral del estado nutricional, detección de hipertensión arterial infantil y factores asociados en niños y niñas escolares de la Escuela Básica "José María BogarínLa Salle" en el año 2017 financiado por la Dirección General de Investigación Científica y Tecnológica 
(DGTIC) de la Universidad Nacional de Asunción y la Fundación Facultad de Ciencias Químicas (FUNDAQUIM).

\section{AGRADECIMIENTOS}

Agradecemos a los estudiantes y colaboradores de la carrera de Bioquímica, Farmacia y Nutrición, de la Facultad de Ciencias Químicas de la Universidad Nacional de Asunción, quienes contribuyeron en la logística y actuaron de auxiliares en la recolección de datos durante el trabajo de campo, en especial a Nathalia Rodríguez, Sonia Ayala, Pamela Mancuello, Antonio Robles, Aníbal Espínola, Nadia Centurión, Mayra Benítez, Edilza Soarez, Mónica Grance, Judith Galeano, Iván Lezcano, Jessica Cantero, María Paz Brun, Lourdes Samaniego, Nilsa Lial, Patricia Vera y todas las personas que contribuyeron de manera significativa en esta investigación.

\section{REFERENCIAS}

1. Pierlot R, Cuevas-Romero E, Rodríguez-Antolín J, Méndez-Hernández P, Martínez-Gómez M. Prevalencia de Síndrome Metabólico en Niños y Adolescentes de América. Tip. 2017; 20(1):40-9. doi: https://doi.org/ 10.1016/j.recqb.2016.11.004

2. Reaven GM. Role of insulin resistance in human disease. Diabetes. 1988;37(12):1595-607. doi: https://doi.org/ 10.2337/diab.37.12.1595

3. Castillo Durán C, Le Roy C, Osorio J. Obesidad y Síndrome Metabólico en Niños y Adolescentes. Rev Med Clin Condes. 2012;23(2):160-4. doi: https://doi.org/ 10.1016/S0716-8640(12)70293-6

4. Eyzaguirre F, Mericq V. Insulin resistance markers in children. Horm Res. 2009;71(2):65-74. doi: https://doi.org/ $10.1159 / 000183894$

5. Palhares HM da C, da Silva AP, Resende DCS, Pereira G de A, Rodrigues V, Borges M de F. Evaluation of clinical and laboratory markers of cardiometabolic risk in overweight and obese children and adolescents. Clinics (Sao Paulo). 2017;72(1):36-43. doi: http://dx.doi.org/ 10.6061/clinics/2017(01)07

6. Higgins V, Adeli K. Pediatric Metabolic Syndrome: Pathophysiology and Laboratory Assessment. Ejifcc. 2017;28(1):25-42.

7. Camhi SM, Katzmarzyk PT. Tracking of cardiometabolic risk factor clustering from childhood to adulthood. Int J Pediatr Obes. 2010;5(2):122-9. doi: http://dx.doi.org/10.3109/17477160903111763

8. Barker DJP. The origins of the developmental origins theory. J Intern Med. 2007;261(5):412-7. doi: http://dx.doi.org/10.1111/j.1365-2796.2007.01809.x
9. Gluckman PD, Buklijas T, Hanson MA. The Developmental Origins of Health and Disease (DOHaD) Concept: Past, Present, and Future. Epigenome Dev Orig Heal Dis. 2015;(April):1-15.

10. Zimmet P, Alberti KGM, Kaufman F, Tajima N, Silink $\mathrm{M}$, Arslanian S, et al. The metabolic syndrome in children and adolescents - an IDF consensus report. Pediatr Diabetes. 2007;8(5):299-306. doi: http://dx.doi.org/10. 1111/j.1399-5448.2007.00271.x

11. D'Adamo E, Santoro N, Caprio S. Metabolic Syndrome in Pediatrics: Old Concepts Revised, New Concepts Discussed. Pediatr Clin North Am. 2011;58(5):1241-55. doi: http://dx.doi.org/10.1016/j.pcl.2011.07.005

12. De Ferranti SD, Gauvreau K, Ludwig DS, Neufeld EJ, Newburger JW, Rifai N. Prevalence of the metabolic syndrome in American adolescents: Findings from the Third National Health and Nutrition Examination Survey. Circulation. 2004;110 (16):2494-7. doi: http://dx.doi.org/ 10.1161/01.CIR.0000145117.40114.C7

13. Vanlancker T, Schaubroeck E, Vyncke $K$, CadenasSanchez C, Breidenassel C, González-Gross M, et al. Comparison of definitions for the metabolic syndrome in adolescents. The HELENA study. Eur J Pediatr. 2017;176 (2):241-52. doi: http://dx.doi.org/10.1007/s00431-016-2831-6

14. Magge SN, Goodman E, Armstrong SC, Committee on nutrition, section on endocrinology, section on obesity. The Metabolic Syndrome in Children and Adolescents: Shifting the Focus to Cardiometabolic Risk Factor Clustering. Pediatrics. 2017;140(2):e20171603. doi: http://dx.doi.org/10.1542/peds.2017-1603 
15. López P, Araujo C, Leguizamón C, Ayala A, Scott C, Maldonado D. Prevalencia de Síndrome Metabólico en Adolescentes con Sobrepeso u Obesidad. Pediatr. (Asunción). 2012;39(1):21-5.

16. Riveros Sasaki K, Alderete Peralta V, Sánchez Bernal S. Frecuencia de elementos del Síndrome Metabólico en adolescentes de un colegio público. Pediatr. (Asunción) Organo Of la Soc Paraguaya Pediatría. 2012;39 (1):13-9.

17. NCEP-ATP. Executive summary of the third report of the national cholesterol education program (ncep) expert panel on detection, evaluation, and treatment of high blood cholesterol in adults (adult treatment panel iii). JAMA. 2001; 285 (19):2486-97. doi: http://dx.doi.org/ 10.1001/jama.285.19.2486

18. Alberti KGMM, Zimmet P, Shaw J. Metabolic syndrome - a new world-wide definition. A Consensus Statement from the International Diabetes Federation. Diabet Med. 2006; 23 (5):469-80. doi: http://dx.doi.org/ 10.1111/j.1464-5491.2006.01858.x

19. Guzmán-Guzmán IP, Salgado-Bernabé AB, Muñoz Valle JF, Vences-Velázquez A, Parra-Rojas I. Prevalence of metabolic syndrome in children with and without obesity. Med Clínica. 2015;144(5):198-203.

20. MSPBS. Unas 2600 muertes al año se asocian a la obesidad en Paraguay [Internet]. 2019 [cited 2019 Abr 27]. Available from: https://www.mspbs.gov.py/portal
21. OMS. Actualización sobre la Comisión para acabar con la obesidad infantil. 68o Asamblea Mundial de la Salud. 2015.

22. Lira M, Vio A. Informe Mapa Nutricional 2015. JUNAEB. Junaeb. 2015.

23. Vio del R F, Lera L, González CG, Fierro MJ, Salinas J. Diagnóstico de la situación alimentaria y nutricional de niños de tercero a quinto año básico de la comuna de la Reina, Santiago de Chile. Rev Chil Nutr. 2017;44 (3):244-50. doi:http://dx.doi.org/10.4067/s0717-75182017000300244

24. Ramírez-Vélez R, Anzola A, Martinez-Torres J, Vivas A, Tordecilla-Sanders A, Benavides D, et al. Metabolic Syndrome and associated factors in a population-based sample of schoolchildren in Colombia: The FUPRECOL Study. Metab Syndr Relat Disord. 2016;14 (9):455-62. doi: http://dx.doi.org/10.1089/met.2016.0058

25. Barbalho SM, Oshiiwa M, Sato Fontana LC, Ribeiro Finalli EF, Paiva Filho ME, Machado Spada AP. Metabolic syndrome and atherogenic indices in school children: A worrying panorama in Brazil. Diabetes Metab Syndr Clin Res Rev. 2017;(2016):10-2. doi: http://dx.doi.org/10.10 16/j.dsx.2017.03.024

26. Pretto ADB, Kaufmann CC, Dutra GF, Albernaz EP. Prevalence of factors associated to metabolic syndrome in a cohort of children in South Brazil. Nutr Hosp. 2015;32 (1):118-23. 